\title{
On the Accuracy of Hyperspherical Harmonics Approaches to Photonuclear Reactions
}

\author{
Nir Barnea ${ }^{1}$, Winfried Leidemann², Giuseppina \\ Orlandini $^{2}$, Victor D. Efros ${ }^{3}$ Edward L. Tomusiak ${ }^{4}$ \\ 1 The Racah Institute of Physics, \\ The Hebrew University, \\ 91904 Jerusalem, Israel \\ ${ }^{2}$ Dipartimento di Fisica, \\ Università di Trento \\ and Istituto Nazionale di Fisica Nucleare, \\ Gruppo Collegato di Trento, \\ I-38050 Povo, (Trento) Italy \\ ${ }^{3}$ Russian Research Centre "Kurchatov Institute", \\ Kurchatov Square, 1, \\ 123182 Moscow, Russia \\ ${ }^{4}$ Department of Physics and Astronomy, \\ University of Victoria, \\ Victoria, BC V8P 1A1, Canada
}

(Dated: August 10, 2018) 


\begin{abstract}
Using the Lorentz Integral Transform (LIT) method we compare the results for the triton total photodisintegration cross section obtained using the Correlated Hyperspherical Harmonics (CHH) and the Effective Interaction Hyperspherical Harmonics (EIHH) techniques. We show that these two approaches, while rather different both conceptually and computationally, lead to results which coincide within high accuracy. The calculations which include two- and three-body forces are of the same high quality in both cases. We also discuss the comparison of the two approaches in terms of computational efficiency. These results are of major importance in view of applications to the much debated case of the four-nucleon photoabsorption.
\end{abstract}

PACS numbers: 21.45.+v, 25.10.+s, 25.20.DC, 27.10.+h 


\section{INTRODUCTION}

In the last few years there has be a resurgence of interest in the photodisintegration of few-nucleon systems. This is essentially due to two reasons. One of them is that few-body theories have now achieved the ability to produce results of high accuracy for reactions into the many-body continuum. The other is the ongoing debate about the existence and nature of three-body forces, which finds a natural testing ground in reactions involving the three-nucleon systems. Some theoretical results on photonuclear reactions with light nuclei using realistic two- and three-body potentials have already been published [? ? ? and among them is a benchmark between the correlated hyperspherical harmonics $(\mathrm{CHH})$ and Faddeev approaches [? ] on the total photodisintegration cross section of the three-body systems. This benchmark demonstrated the high degree of accuracy reached by these two techniques. At the same time it showed the inadequacy of existing experimental data to allow any conclusion about the three-nucleon force effect, whose magnitude turns out to be smaller than the rather large error bars.

In this work we test the EIHH method on the triton photonuclear cross section with realistic forces. This method is also based on a hyperspherical harmonics expansion, but uses the concept of an effective interaction to speed up the convergence of the expansion. We believe that it is necessary to benchmark EIHH results on a realistic force calculation. In fact, when combined with the LIT method [? ], this approach is very promising (see e.g. [? ? ]) for the study of electromagnetic reactions on heavier systems, where three-body force effects might be more prominent.

In the following we first briefly recall the LIT method (Section II). In Section III the hyperspherical expansion method is summarized, together with the $\mathrm{CHH}$ (Section 【AI) and EIHH (Section [IIB) techniques. They address the problem of convergence for the solutions

of the Schrödinger and Schrödinger-like equations required by the LIT method. Section IV] contains results for the triton photoabsorption with a realistic two- and three-nucleon force and, in view of applications to the electromagnetic responses of heavier systems, also a discussion about the computational efficiency of the two approaches. 


\section{THE LIT METHOD}

The LIT method has been described extensively in several publications [? ? ]. Here we only recall the key points.

The calculation of any inclusive electromagnetic cross section requires the knowledge of the response function

$$
R(\omega)=y_{n}|\langle 0|\Theta| n\rangle|^{2} \delta\left(\omega-E_{n}+E_{0}\right)
$$

where $\omega$ represents the energy transferred by the elctromagnetic probe, $|0\rangle$ and $E_{0}$ are ground state wave function and energy of the system undergoing the reaction, $|n\rangle$ and $E_{n}$ denote eigenstates and eigenvalues of the Hamiltonian $H$, and $\Theta$ is the operator relevant to the reaction. The relation between the total photoabsorption cross section and the response function is given by

$$
\sigma_{\gamma}(\omega)=4 \pi^{2} \alpha \omega R(\omega)
$$

where $\alpha$ is the fine structure constant and $\Theta$ of Eq. (11) is the nuclear current operator. In the low-energy region considered here one can rely on Siegert's theorem and use for $\Theta$ the unretarded dipole operator

$$
\Theta=\sum_{i}^{A} z_{i} \tau_{i}^{3} .
$$

The LIT method consists in calculating $R(\omega)$ in three steps. Step 1. The equation

$$
\left(H-E_{0}-\omega_{0}+i \Gamma\right)|\tilde{\Psi}\rangle=\Theta|0\rangle
$$

is solved for many $\omega_{0}$ and a fixed $\Gamma$.

This is a Schrödinger-like equation with a source. It can be shown easily that the solutions $|\tilde{\Psi}\rangle$ are localized. Thus one only needs a bound state technique to obtain them. In particular one can adopt the same bound state technique as for the solution of the ground state, which is an input for Eq. (41).

The values of the parameters $\omega_{0}$ and $\Gamma$ are chosen in relation to the physical problem. In fact, as will become clear in Step 2, the value of $\Gamma$ is a kind of "energy resolution" for the response function and the values of $\omega_{0}$ scan the region of interest. In our case of triton photodisintegration we solve Eq. (4) with $\Gamma=10$ and $20 \mathrm{MeV}$ and for a few hundred of $\omega_{0}$ values chosen in the interval $-40 \mathrm{MeV}$ to $200 \mathrm{MeV}$. 
Step 2. The overlaps $\langle\tilde{\Psi} \mid \tilde{\Psi}\rangle$ of the solutions from Step 1. are calculated. Of course these overlaps depend on $\omega_{0}$ and $\Gamma$. A theorem for integral transforms based on the closure property of the Hamiltonian eigenstates [? ] ensures that in the present case this dependence can be expressed as [? ]

$$
\mathrm{L}\left(\omega_{0}, \Gamma\right)=\langle\tilde{\Psi} \mid \tilde{\Psi}\rangle=\int R(\omega) \mathcal{L}\left(\omega, \omega_{0}, \Gamma\right) d \omega
$$

where $\mathcal{L}$ is the Lorentzian function centered at $\omega_{0}$ and with $\Gamma$ as a width:

$$
\mathcal{L}\left(\omega, \omega_{0}, \Gamma\right)=\frac{1}{\left(\omega-\omega_{0}\right)^{2}+\Gamma^{2}}
$$

Therefore by solving Eq. (4) one can easily obtain the Lorentz integral transform of the response function.

Step 3. The transform (5) is inverted (see e.g. Ref. [? ? ]) in order to obtain the response function and with it the cross section. Of course the inversion result has to be independent of $\Gamma$ and show a high degree of stability.

\section{THE HH EXPANSION TECHNIQUE}

In the $\mathrm{HH}$ expansion approach the basis functions for the solution of the $A$-body Schrödinger equation for the ground state $|0\rangle$, as well as of the $A$-body "LIT equation" (4) for $|\tilde{\Psi}\rangle$, are expressed as functions of the hyperspherical coordinates. These are constructed starting from the $A-1$ Jacobi coordinates $\vec{\xi}_{i}$ for the $A$-particle system. They consist of a hyperradius $\rho_{A-1}=\sqrt{\sum_{i}^{A-1}\left(\vec{\xi}_{i}\right)^{2}}$ and a hyperangle $\Omega_{A-1}=\left\{\hat{\xi}_{1}, \hat{\xi}_{2}, \cdots, \hat{\xi}_{A-1}, \varphi_{2}, \varphi_{3}, \cdots, \varphi_{A-1}\right\}$ $=\left\{\Omega_{A-2}, \varphi_{A-1}, \hat{\xi}_{A-1}\right\}$, where the $A-2$ hyperangles $\left\{\varphi_{2}, \varphi_{3}, \cdots, \varphi_{A-1}\right\}$ are defined in the $[0, \pi / 2]$ interval through the relation

$$
\sin \varphi_{n}=\xi_{n} / \rho_{n}, \quad n=2,3, \cdots, A-1
$$

In terms of these coordinates the Laplace operator for $n$ Jacobi coordinates is

$$
\Delta_{n}=\frac{1}{\rho_{n}^{3 n-1}} \frac{\partial}{\partial \rho_{n}} \rho_{n}^{3 n-1} \frac{\partial}{\partial \rho_{n}}-\frac{1}{\rho_{n}^{2}} \hat{K}_{n}^{2},
$$

i.e. a sum of a hyperradial operator and of a term containing the hyperspherical, or grand angular momentum operator $\hat{K}_{n}^{2}$. The latter can be expressed recursively in terms of the 
hyperangular momentum operator associated with the subset $\left\{\vec{\xi}_{1}, \vec{\xi}_{2}, \cdots, \vec{\xi}_{n}\right\}, \hat{K}_{n-1}^{2}$, and $\hat{\ell}_{n}^{2}$, the orbital momentum operator corresponding to the $n$th Jacobi coordinate, namely [? ]:

$$
\hat{K}_{n}^{2}=-\frac{\partial^{2}}{\partial \varphi_{n}^{2}}+\frac{3 n-6-(3 n-2) \cos 2 \varphi_{n}}{\sin 2 \varphi_{n}} \frac{\partial}{\partial \varphi_{n}} \frac{1}{\cos ^{2} \varphi_{n}} \hat{K}_{n-1}^{2}+\frac{1}{\sin ^{2} \varphi_{n}} \hat{\ell}_{n}^{2} .
$$

In the above equation $\hat{K}_{1}^{2} \equiv \hat{\ell}_{1}^{2}$, and the angular momentum operator associated with these $n$ coordinates is $\overrightarrow{\hat{L}}_{n}=\overrightarrow{\hat{L}}_{n-1}+\overrightarrow{\hat{\ell}}_{n}$. The operators $\hat{K}_{n}^{2}, \hat{\ell}_{n}^{2}, \hat{K}_{n-1}^{2}, \hat{L}_{n}^{2}$ and $\hat{L}_{n}^{(z)}$ commute with each other.

Given the set of hyperspherical coordinates, the hyperspherical harmonics functions $\mathcal{Y}_{\left[K_{n}\right]}$ are the eigenfunctions of the hyperangular momentum operator $\hat{K}_{n}^{2}$,

$$
\hat{K}_{n}^{2} \mathcal{Y}_{\left[K_{n}\right]}=K_{n}\left(K_{n}+3 n-2\right) \mathcal{Y}_{\left[K_{n}\right]},
$$

where $K_{n}$ is the hyperangular quantum number and the symbol $\left[K_{n}\right]$ stands for the set of quantum numbers $\left\{K_{n}, K_{n-1}, \cdots, K_{2} ; L_{n}, M_{n} ; L_{n-1}, \cdots, L_{2} ; \ell_{n}, \ell_{n-1}, \cdots, \ell_{1}\right\}$. The explicit expression for the $\mathrm{HH}$ functions of $n$ Jacobi coordinates can be constructed recursivly as described in Ref. [? ].

The HH functions $\mathcal{Y}_{\left[K_{n}\right]}$ form a complete and orthonormal set of functions that satisfy

$$
\left\langle\mathcal{Y}_{\left[K_{n}\right]} \mid \mathcal{Y}_{\left[K_{n}^{\prime}\right]}\right\rangle=\delta_{\left[K_{n}\right],\left[K_{n}^{\prime}\right]}
$$

The particle permutation properties of the $\mathrm{HH}$ functions can be implemented in different ways. In the $\mathrm{CHH}$ approach used here this is done as described in Ref. [? ], while in the EIHH framework two recently developed powerful algorithms are employed [? ? ].

As functions of the hyperspherical coordinates the antisymmetric spin-isospin configuration space basis functions are written in the form

$$
\mathcal{H} \mathcal{H}_{N[K, \gamma]}^{[f]}\left(\rho, \Omega_{A-1}, \sigma, \tau\right)=R_{N}(\rho) \Gamma_{[K, \gamma]}^{[f]}(\Omega, \sigma, \tau)
$$

where, for the hyperradial part of the wave function one chooses an orthonormalized set of functions, $R_{N}(\rho) \sim L_{N}^{\nu}\left(\rho / \rho_{0}\right) \exp \left(-\rho / 2 \rho_{0}\right)$. Here $L_{N}^{\nu}$ are the generalized Laguerre polynomials, and $\nu$ and $\rho_{0}$ are free parameters [? ]. In the fully antisymmetric functions $\Gamma_{[K, \gamma]}^{[f]}$ the notation $[K, \gamma]$ stands for the set of quantum numbers $\left\{K, J, M_{J}, L, S, T, M_{T}\right\}$, i.e. total angular momentum and its third component, orbital angular momentum, spin, total isospin and isospin projection of the A-body system, respectively and $\sigma, \tau$ indicate collectively the spin and isospin variables of the $A$ nucleons. These functions are obtained by the coupling of 
the HH functions $\mathcal{Y}_{[K]}^{[f] \mu_{f}}(\Omega)$ with specified $L$ and $M$ of the representation $[f]$ and the spin-

isospin functions $\Theta_{S M_{S} T M_{T}}^{[\bar{f}] \mu_{f}}(\sigma, \tau)$ with specified $S, M_{S}, T$ and $M_{T}$ of the conjugate Young scheme $[\bar{f}]$. In many cases the rate of convergence of the $\mathrm{HH}$ expansion is rather slow. In order to accelerate it one can follow two rather different approaches, leading to the $\mathrm{CHH}$ and EIHH methods.

\section{A. The CHH Approach}

The CHH approach [? ] has been used extensively in the past. While the underlying idea is common, there exist a number of slightly different versions of this approach. In the following we describe how the $\mathrm{CHH}$ has been applied by the present authors in this and previous works [? ? ].

The main idea of the $\mathrm{CHH}$ approach consists of the insertion in Eq. (12) of a Jastrow factor $\mathcal{J}$ embodying the short range correlation due to the repulsive part of the potential. Such a repulsion leads to high momentum components in the wave function which make the convergence of the $\mathrm{HH}$ expansion rather slow. Therefore one modifies Eq. (12) into

$$
\mathcal{C H} \mathcal{H}_{N[K, \gamma]}^{[f]}\left(\rho, \Omega_{A-1}, \sigma, \tau\right)=\mathcal{J} R_{N}(\rho) \Gamma_{[K, \gamma]}^{[f]}(\Omega, \sigma, \tau)
$$

Here $\mathcal{J}$ is taken as a state-dependent correlation operator of the form

$$
\mathcal{J}=\mathcal{S} \prod_{i<j} \sum_{s, t} f_{s, t}\left(r_{i j}\right) \hat{P}_{s, t}(i j)
$$

where $\hat{P}_{s, t}(i j)$ are projection operators onto nucleon pairs $(i j)$ with spin $s$ and isospin $t$ and $\mathcal{S}$ is a particle symmetrization operator. The radial parts of the correlation operator are chosen as follows. For $r_{i j}<r_{0}$ (the healing distance) $f_{s, t}\left(r_{i j}\right)$ is chosen to be the zero energy pair wave function in the corresponding $s, t$ state. The healing is insured by imposing the condition $f_{s, t}\left(r_{i j}\right)=1$ for $r_{i j}>r_{0}$ and $f_{s, t}^{\prime}\left(r_{i j}\right)=0$ for $r_{i j}=r_{0}$. The $s, t=1,3$ and $s, t=3,1$ cases are determined from the ${ }^{1} S_{0}$ and ${ }^{3} S_{1}$ partial wave of the NN potential. Since for the $s, t=1,1$ and $s, t=3,3$ cases the ${ }^{1} P_{1}$ and ${ }^{3} P_{1}$ potentials are not sufficiently attractive to obtain a healing distance, we introduce an additional intermediate range central interaction. Further details can be found in [? ]. 


\section{B. The EIHH approach}

In contrast to the $\mathrm{CHH}$ approach, which focuses on the short range properties of the wave functions, the EIHH approach focuses on the potential operator. The acceleration of the convergence is obtained by introducing a two-body effective interaction $V_{\text {eff }}^{[2]}[?$ ? ]. This is done by the division of the total $\mathrm{HH}$ space in $\mathrm{P}$ and $\mathrm{Q}$ spaces realized via the $\mathrm{HH}$ quantum number $K$ (P space: $K \leq K_{\max }$, Q space: $\left.K>K_{\max }\right)$. Since in hyperspherical coordinates the total Hamiltonian is written as

$$
H=\frac{1}{2 m}\left(-\Delta_{\rho}+\frac{\hat{K}^{2}}{\rho^{2}}\right)+\sum_{i<j} V_{i j},
$$

one can extract from it a "two-body" Hamiltonian of particles $A$ and $(A-1)$

$$
H_{2}(\rho)=\frac{1}{2 m} \frac{\hat{K}^{2}}{\rho^{2}}+V_{A,(A-1)},
$$

which, however, contains the hyperspherical part of the A-body kinetic energy. Since the HH functions of the $(A-2)$ system are eigenfunctions of $\hat{K}_{A-2}^{2}$ one has an explicit dependence of $H_{2}$ on the quantum number $K_{A-2}$ of the residual system, i.e. $H_{2} \rightarrow H_{2}^{K_{A-2}}$. Applying the hermitian version of the Lee-Suzuki method [? ? ? ] to $H_{2}$ one gets an effective Hamiltonian $H_{2 e f f}$. The effective interaction $V_{e f f}^{[2]}$ is obtained from

$$
V_{e f f}^{[2] K_{A-2}}(\rho)=H_{2 e f f}^{K_{A-2}}(\rho)-\frac{1}{2 m} \frac{\hat{K}^{2}}{\rho^{2}} .
$$

This $V_{\text {eff }}^{[2]}$ replaces $V_{i j}$ in Eq. (15) when one projects the solution on the $\mathrm{P}$-space. This effective potential has the following property: $V_{e f f}^{[2]} \rightarrow V_{i j}$ for $P \rightarrow 1$. Due to the "effectiveness" of the operator the solution of the Schrödinger equation converges faster to the true one. The present $\mathrm{HH}$ formulation of an effective potential has the following peculiarities, which make it particularly "effective": (i) $V_{e f f}^{[2]}$ is $\rho$ dependent, therefore it contains information on the "medium"; (ii) because of the above mentioned $K_{A-2}$ dependence the (A-2) residual system is not a pure spectator and its state influences the state of the pair, therefore $V_{e f f}^{[2]}$ can be viewed as a state dependent effective interaction. Moreover we point out that the effective potential can be interpreted as a kind of momentum expansion, since the short range resolution is increased with growing $K_{\max }$. 


\section{RESULTS}

We have compared the transform $\mathrm{L}\left(\omega_{0}, \Gamma\right)$ and response function $R(\omega)$ as computed by the $\mathrm{CHH}$ and EIHH approaches. The potentials employed were the AV18 [? ] two-body and UIX [? ] three-body potential models. In the case of $\mathrm{CHH}$ we expand the ground state with 11 hyperradial functions and the hyperspherical states with $K$ up to $K_{\max }=10$, but consider additional selected states up to $K_{\max }=100$. For the $\mathrm{T}=1 / 2$ component of $|\tilde{\Psi}\rangle$ we take 26 hyperradial functions, $K_{\max }=9$ and additional selected states up to $K_{\max }=65$. For $\mathrm{T}=3 / 2 K_{\max }=15$ and 26 hyperradial functions are sufficient for a convergent result. In the case of EIHH we expand with 26 hyperradial functions up to $K_{\max }=14$ (ground state) and $K_{\max }=15(|\tilde{\Psi}\rangle)$. In Fig. 1 the EIHH rate of convergence of $\mathrm{L}\left(\omega_{0}, \Gamma\right)$ is shown for the $(\mathrm{J}=1 / 2, \mathrm{~T}=1 / 2)$ contribution to $\tilde{\Psi}$ with $\Gamma=10 \mathrm{MeV}$. One sees that the convergence is very good in the whole energy range. One also notes that small oscillations are present in the transform at higher energies. If needed one could improve the result by increasing the number of hyperradial states. Convergence patterns of the other $(J, T)$ contributions in the EIHH calculation are similar to that shown in Fig. 1. Also the rate of convergence of the $\mathrm{CHH}$ calculation for $\mathrm{T}=3 / 2$ is rather similar to that of $\mathrm{Fig}$. 1 while for $\mathrm{T}=1 / 2$ the convergence is considerably slower. Comparable differences in the rate of convergence of the two methods have also been found for the ${ }^{4}$ He total photoabsorption cross section with semirealistic potential models [? ]. Thus, in view of applications to more complex nuclei, the EIHH method seems to be preferable from the calculational point of view due to its faster convergence. In Fig. $2 \mathrm{a}$ we show the results for the total Lorentz integral transform of $R(\omega)$ (contributions of $\mathrm{J}=1 / 2$ and $\mathrm{J}=3 / 2$ components are summed) for both the $\mathrm{T}=1 / 2$ and $\mathrm{T}=3 / 2$ cases and with $\Gamma=20 \mathrm{MeV}$. It is evident that for both isospin channels the agreement is excellent over the whole considered energy range. In Fig. 2b the relative difference $\Delta \mathrm{L}=\left(\mathrm{L}^{\mathrm{EIHH}}-\mathrm{L}^{\mathrm{CHH}}\right) / \mathrm{L}^{\mathrm{CHH}}$ is shown in percent. It is readily seen that $\Delta \mathrm{L}$ does not exceed $0.4 \%$ in the peak region. For higher energies the differences can become somewhat larger but remain below $2 \%$ up to pion threshold. In Fig. 3a we show the total photoabsorption cross section. The results of the two approaches remain very close also after inverting the transforms (Step 3 in Section III). In fact the EIHH and CHH curves are hardly distinguishable. Even if the agreement has slightly deteriorated compared to Fig. 2 due to the inversion procedure, the quality remains excellent. As shown in Fig. 3]b 
the difference remains below $0.8 \%$ in the peak region and has a maximum of $2.4 \%$ up to pion threshold. We would like to point out that the agreement of the two $\mathrm{HH}$ calculations in the peak region is even better than the already good agreement of $\mathrm{CHH}$ and Faddeev calculations shown in the above mentioned benchmark [? ]. We do not compare the present results with the Faddeev cross section in [? ] since that benchmark was made neglecting the charge dependent terms in the AV18 nuclear force. Though that effect is small (of the order of $1 \%$ in the peak region), it is much larger than the difference between $\mathrm{CHH}$ and $\mathrm{EIHH}$ calculations. If one considers that EIHH and $\mathrm{CHH}$ approaches differ substantially both from the conceptual and calculational points of view (of course completely independent codes are used), the agreement of the results is really remarkable and shows that one can rely on the application of the EIHH approach to heavier systems with a high degree of confidence. In particular, its application to the calculation of the total photonuclear cross section of ${ }^{4} \mathrm{He}$ within realistic nuclar force models, containing two- and three-body forces, would be very much needed. It could shed some light on the role of three-nucleon forces, which might be even more important than already found in the three-nucleon system. Moreover, it would make a valuable contribution to the clarification of the present rather confused situation in the comparison between theoretical and experimental results in ${ }^{4} \mathrm{He}$ (see e.g. [? ? ]). Work in this direction is in progress.

\section{Acknowledgment}

This work was supported by the grant COFIN03 of the Italian Ministery of University and Research. V.D.E. acknowledges support from the RFBR, grant 05-02-17541. The work of N.B. was supported by the ISRAEL SCIENCE FOUNDATION while E.L.T. was supported by the National Science and Engineering Research Council of Canada. (grant no. 202/02). 
Fig. 1

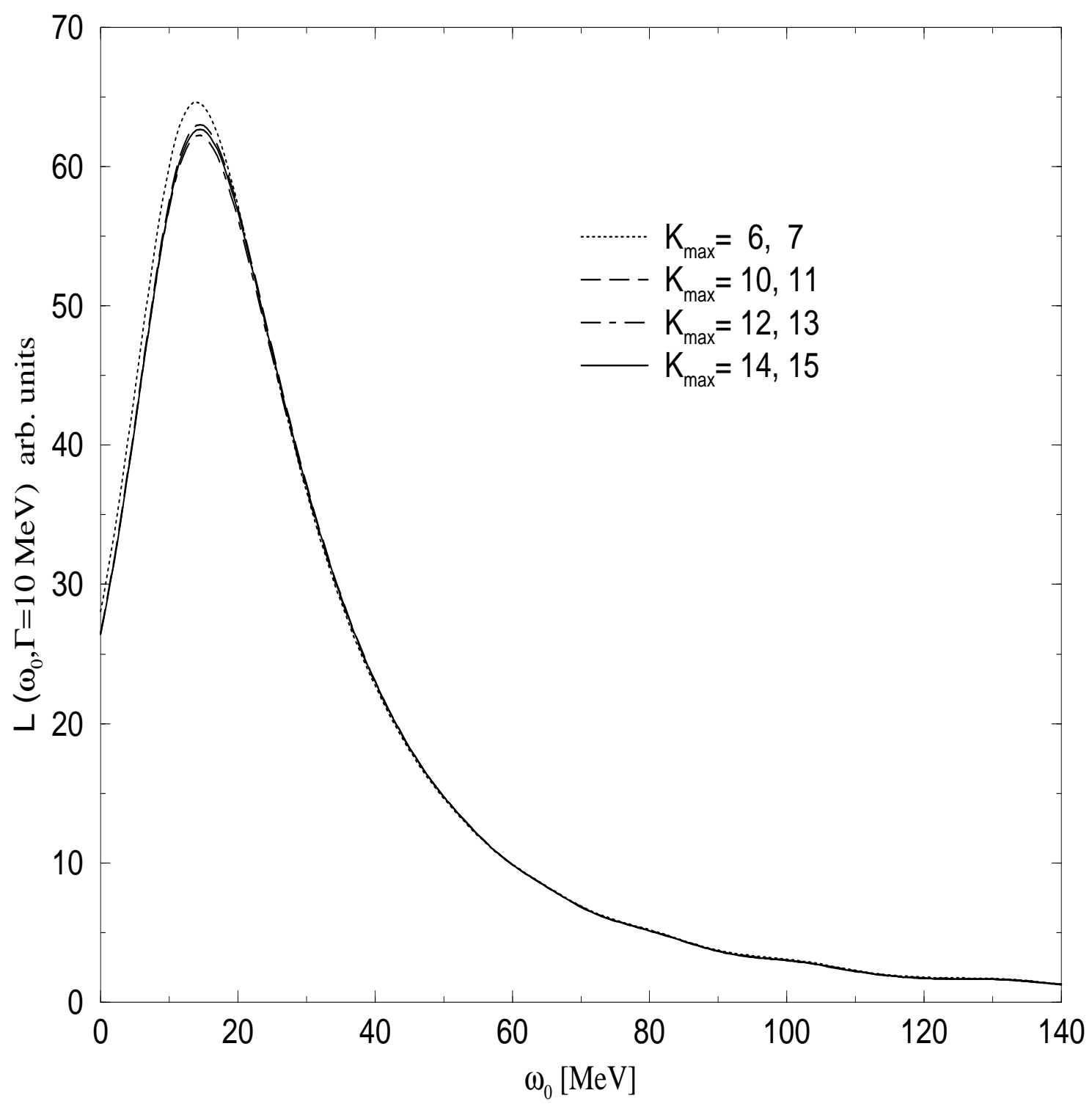

FIG. 1: Rate of EIHH convergence of the LIT of the unretarded dipole response function (Eq.(5)) for $\Gamma=10 \mathrm{MeV}$, limited to the $(\mathrm{J}=1 / 2, \mathrm{~T}=1 / 2)$ component. The even values of $K_{\max }$ refer to the calculation of the ground state $|0\rangle$, the odd values to that of the Lorentz state $\tilde{\Psi}$. 
Fig. 2
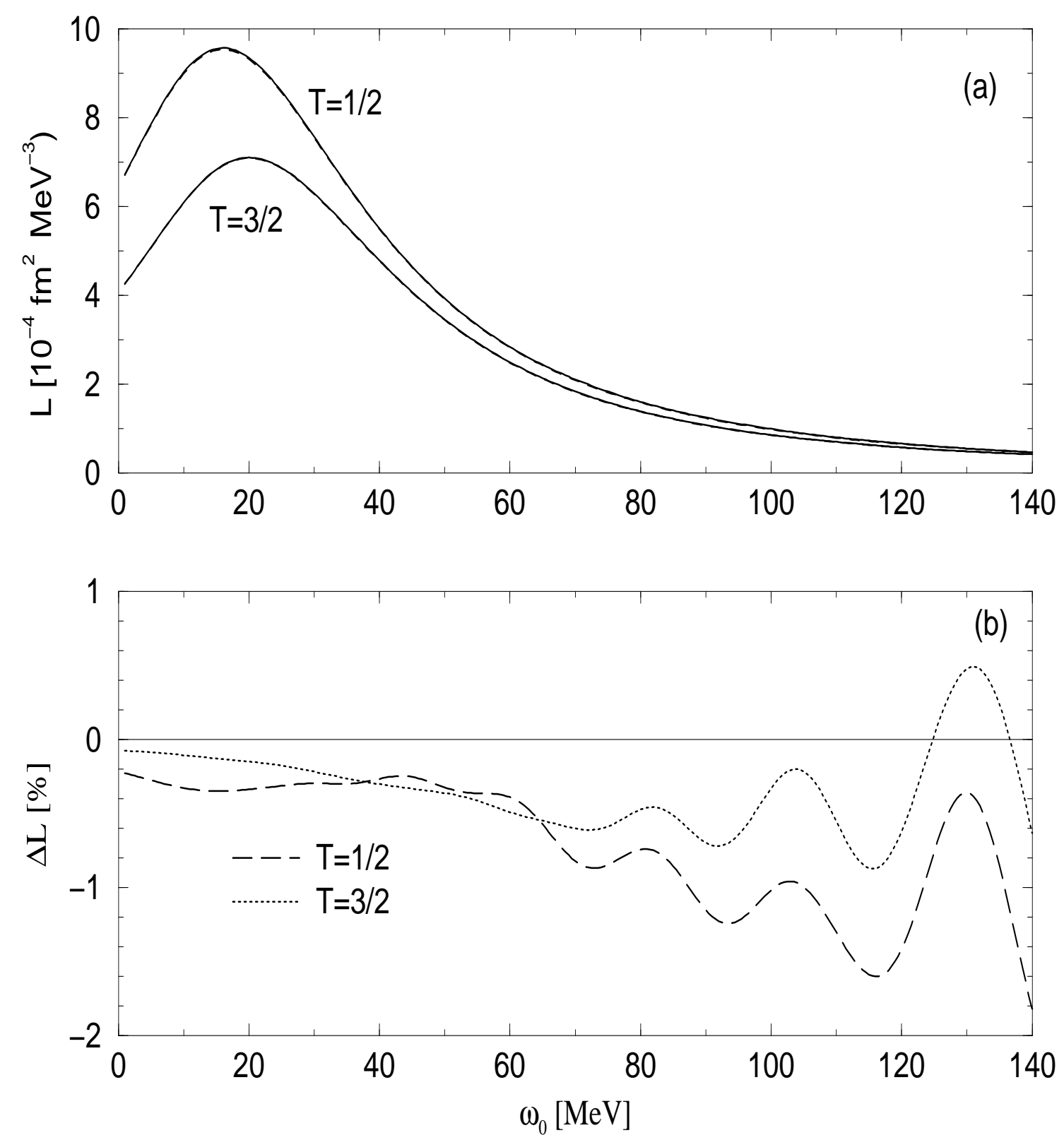

FIG. 2: Comparison between CHH and EIHH results for the LIT of the unretarded dipole response function (Eq.(5)) in the two isospin channels. (a): CHH (full curve); EIHH (dashed curve). (b): The difference $\Delta \mathrm{L}=\left(\mathrm{L}^{\mathrm{EIHH}}-\mathrm{L}^{\mathrm{CHH}}\right) / \mathrm{L}^{\mathrm{CHH}}$ between the $\mathrm{CHH}$ and EIHH results of Fig. 2a expressed in percent. 
Fig. 3
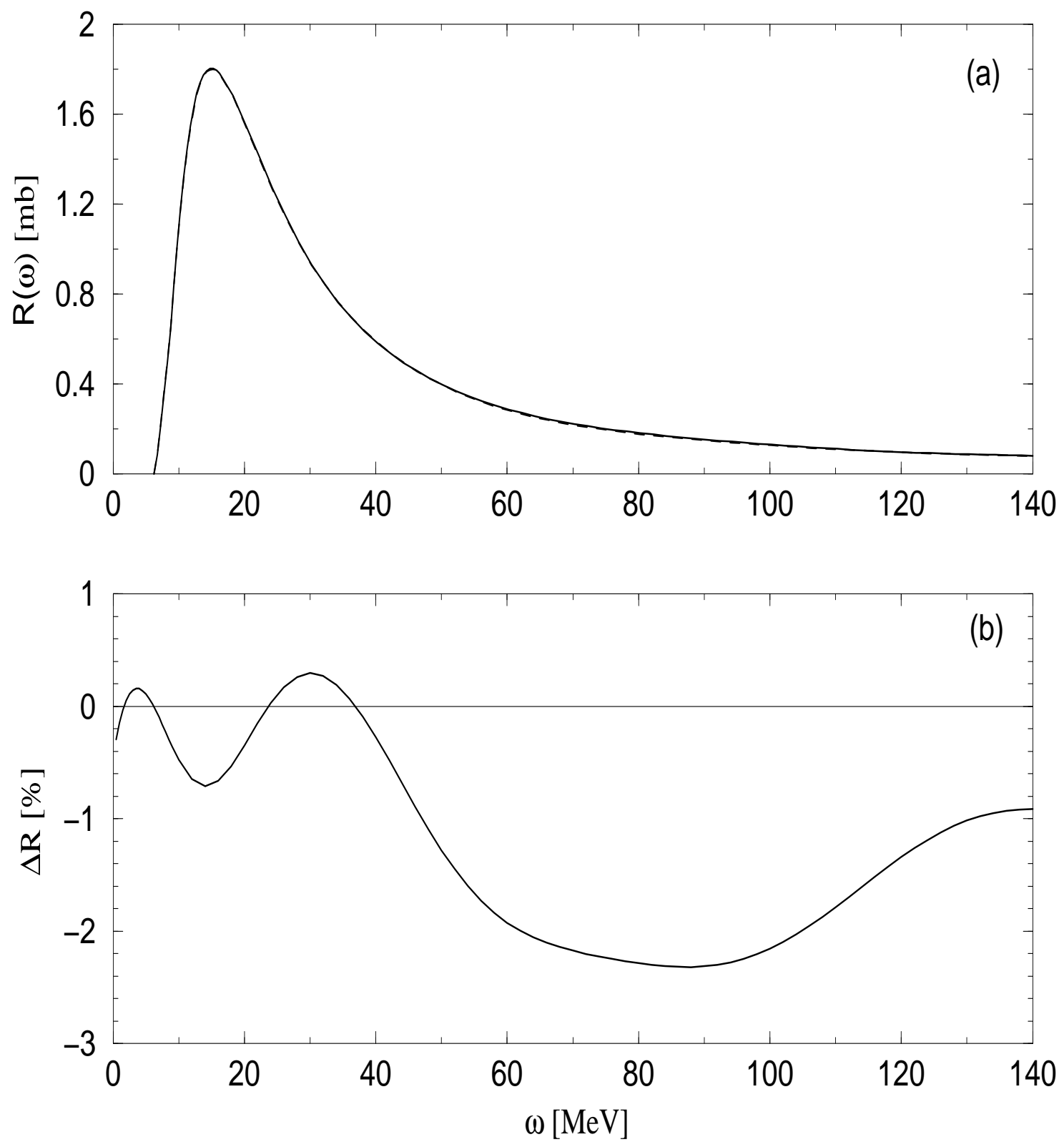

FIG. 3: Comparison between CHH and EIHH total photonuclear cross sections of triton. (a): $\mathrm{CHH}$ (full curve); EIHH (dashed curve), (b): The difference $\Delta R=\left(R^{\mathrm{EIHH}}-R^{\mathrm{CHH}}\right) / R^{\mathrm{CHH}}$ between the CHH and EIHH results of Fig. 3a expressed in percent. 